\title{
Polymorphism at a Sex-Linked Transcription Cofactor in European Tree Frogs (Hyla arborea): Sex-Antagonistic Selection or Neutral Processes?
}

\author{
Christophe Dufresnes $\cdot$ Emilien Luquet $\cdot$ \\ Sandrine Plenet $\cdot$ Matthias Stöck $\cdot$ Nicolas Perrin
}

Received: 26 November 2010/ Accepted: 26 February 2011/Published online: 10 March 2011

(C) Springer Science+Business Media, LLC 2011

\begin{abstract}
Nascent sex chromosomes offer a unique opportunity to investigate the evolutionary fate of genes recently trapped in non-recombining segments. A housekeeping gene (MED15) was recently shown to lie on the nascent sex-chromosomes of the European tree frog (Hyla arborea), with different alleles fixed on the $\mathrm{X}$ and the $\mathrm{Y}$ chromosomes. Here we document a polymorphism (glutamine deletion) in the $\mathrm{X}$ copy of the gene, and use population surveys and experimental crosses to test whether this polymorphism is neutral or maintained by sex-antagonistic selection. Tadpoles from parents of known genotypes revealed significant discrepancies from Mendelian inheritance, suggesting possible sex-antagonistic effects under laboratory conditions. Quantitatively, however, these effects did not meet the conditions for polymorphism maintenance. Furthermore, field estimates of female genotypic frequencies did not differ from Hardy-Weinberg equilibrium and allelic frequencies on the $\mathrm{X}$ chromosome did not differ between sexes. In conclusion, although sexantagonistic effects cannot be excluded given the laboratory conditions, the X-linked polymorphism under study appears neutral in the wild. Alternatively, sex-antagonistic selection might still account for the fixation of a malespecific allele on the $\mathrm{Y}$ chromosome.
\end{abstract}

C. Dufresnes $(\bowtie) \cdot$ M. Stöck $\cdot$ N. Perrin

Department of Ecology and Evolution, University of Lausanne,

1015 Lausanne, Switzerland

e-mail: christophe.dufresnes@unil.ch

E. Luquet $\cdot$ S. Plenet

CNRS UMR 5023, Ecologie des Hydrosystèmes Fluviaux,

Université Claude Bernard Lyon1, 69622 Villeurbanne, France
Keywords Amphibians - Hyla arborea $\cdot$ Glutamine repeats - Population genetics - Sex chromosomes · Transcription cofactor MED15

$\begin{array}{ll}\text { Abbreviations } \\ \text { HWE } & \text { Hardy-Weinberg equilibrium } \\ \text { polyQ } & \text { Poly-glutamine } \\ \text { NS } & \text { Non significant }\end{array}$

\section{Introduction}

Sex chromosome evolution often involves the progressive differentiation of a non-recombining segment in the heterogametic sex (e.g. the XY male in mammals, the WZ female in birds). The absence of recombination can preserve epistatic interactions with sex-specific effects, which may occur either because sex determination depends on several genes [in which case recombination would produce intersex individuals with lowered fecundity; (Charlesworth 1991, 2002; Haldane 1922; Nei 1969)], or because sex differentiation involves genes with sex-antagonistic effects [in which case recombination would induce segregation loads (Rice 1987)].

The absence of recombination has strong consequences for the genes that happen to be trapped in the differential segment. Such genes might acquire a sex-specific sub- or neo-functionalization, with different alleles being expressed and selected for in the heterogametic sex. Alternatively, being kept in a state of permanent heterozygosity, such genes might accumulate deleterious mutations with sufficiently small effects under the combined effect of enhanced drift, background selection and selective sweeps (Charlesworth and Charlesworth 2000; Steinemann and Steinemann 2005). Several genes trapped in the recent 
Y-specific region of medaka fish have already become nonfunctional (Nanda et al. 2002). This progressive decay is responsible for the drastic alterations observed in ancient $\mathrm{Y}$ chromosomes, which have lost most of their functional genes except for a few ones with sex-specific effects. The human Y chromosome, for instance, only retains about one hundred functional genes, many of which are expressed only in testes.

Fully-fledged sex chromosomes are well documented, but, except for a few recently investigated cases (e.g. Bachtrog 2004; Charlesworth 2004; Filatov et al. 2000; Kondo et al. 2004; Liu et al. 2004; McAllister and Charlesworth 1999; Peichel et al. 2004), we know little about young sex chromosomes, which have the potential to provide important insights into the evolutionary fate of genes recently trapped in non-recombining regions. Does the $\mathrm{Y}$ copy rapidly decay under the accumulation of deleterious mutations, or does it develop new, male-specific functions in relation to sex determination or sex differentiation?

A key cofactor (MED15) from the Mediator transcription complex was recently shown to lie on the nonrecombining segment of the nascent sex chromosomes of the European tree frog Hyla arborea (Linnaeus, 1758) (Niculita-Hirzel et al. 2008). Screening populations from Western Switzerland with the Ha5-22 primers (Arens et al. 2000) showed allele 230 to be fixed on the proto-X, while allele 236 was fixed on the proto-Y (Berset-Brändli et al. 2006; 2007). Sequencing both alleles allowed identifying three frame-preserving indels (eight amino acids in total) within the central glutamine-rich (poly-Q) region, with no sign of decay in the $\mathrm{Y}$ copy. A c-DNA analysis further showed both alleles to be expressed during male embryonic development (Niculita-Hirzel et al. 2008).

Changes in glutamine chain lengths have often been assigned functional consequences on various genes (e.g. Buchanan et al. 2004; Gerber et al. 1994; Hancock et al. 2001; Shimohata et al. 2000). Glutamine chains presumably act as polar zippers, forming hydrogen bonds with complementary polar chains (Perutz et al. 1994). Changes in repeat numbers have the potential to change affinities with specific complementary proteins or induce affinities for other regulatory proteins (Perutz et al. 1994). The sexlinked polymorphism documented in $H$. arborea might thus affect the conformation of the Mediator transcription complex, representing the first steps towards the acquisition of male-specific function. However, glutamine repeats are also known to undergo high rates of slippage and rapid evolution (Mularoni et al. 2007), so that these sex differences might actually result from an ancestral neutral polymorphism, with alleles 230 and 236 fixed by chance on the proto $\mathrm{X}$ and proto $\mathrm{Y}$ when recombination stopped.
Here we document a MED15 polymorphism on Hyla X chromosomes in populations from eastern France, where a new Ha5-22 allele (227) was found to segregate with allele 230. Cloning and sequencing showed that allele 227 differs from allele 230 by one glutamine deletion (see Results). Given the possible functional significance of glutamine polymorphisms, the question arises whether this polymorphism is neutral, or maintained by balancing selection. Balanced polymorphism could be maintained through a heterozygote advantage in females. Alternatively, polymorphism may arise from sex-antagonistic effects, if the $\mathrm{X}$ allele 227 is favored in males but disfavored in females, or vice versa (Rice 1984). Theory predicts that sexually antagonistic loci are more likely to remain polymorphic on the sex chromosomes (Rice 1984; Rice and Chippindale 2001), and empirical work indeed suggests that polymorphic sexually antagonistic traits are often located on the $\mathrm{X}$ chromosome in Drosophila (Rice and Chippindale 2001; Gibson et al. 2002).

To address this question, we first tested whether allele frequencies on the $\mathrm{X}$ chromosome differed between adult males and females (as expected in case of sex-antagonistic effect on fitness), and whether genotype frequencies in adult females differed from Hardy-Weinberg (HW) equilibrium (as expected under heterozygote advantage). We then performed within-population crosses between adults with different genotypes, and compared the fitness of offspring differing in their MED15 genotypes.

\section{Methods}

Adult tree frogs were collected during the breeding season (late April to late May) from two populations in the Grésivaudan Region (eastern France): Cheylas $\left(5^{\circ} 59^{\prime} 48^{\prime \prime} \mathrm{E}\right.$, $\left.45^{\circ} 22^{\prime} 53^{\prime \prime} \mathrm{N}\right)$ in 2007 and 2008, and Laissaud, $\left(6^{\circ} 3^{\prime} 51^{\prime \prime} \mathrm{E}\right.$, $\left.45^{\circ} 28^{\prime} 18^{\prime \prime} \mathrm{N}\right)$ in 2008 . Buccal cells were sampled with sterile cotton swabs (Broquet et al. 2007; Pidancier et al. $2003)$, dried with Silicagel, and frozen dry $\left(-80^{\circ} \mathrm{C}\right)$ to be subsequently genotyped for $\mathrm{Ha5}-22$ according to BersetBrändli et al. (2007). Mating pairs were formed randomly without prior information on adult genotypes, and settled in plastic tanks $(17.5 \times 33 \times 18.5 \mathrm{~cm})$ filled with pond water and aquatic plants. Clutches obtained within $24 \mathrm{~h}$ were collected and carried to the laboratory. After hatching, tadpoles were maintained at $22.5^{\circ} \mathrm{C}$ in tanks with water oxygenated by air pumps, a 12:12 h light-dark cycle, and fed daily ad libitum with spinach. A set of fully grown tadpoles (Gosner stage 30-36; Gosner 1960) from 44 pairs chosen according to parental genotypes (see Results) were genotyped for Ha5-22 (tail tips). To estimate whether selection on specific genotypes occurs during larval life, we calculated discrepancies from Mendelian expectations as: 
$\Delta_{I J}=\frac{O_{I J}-E_{I J}}{E_{I J}}$,

where $O_{I J}$ is the observed frequency of genotype $I J$, and $E_{I J}$ the frequency expected from parental genotypes.

A subset of tadpoles that died before metamorphosis was stored in ethanol for genetic analyses, to be used to test for possible genotypic effects on survival. After metamorphosis (stage 46; Gosner 1960), an additional subset of froglets was genotyped (buccal swabs), and their body condition measured as the residuals of a log-log regression of wet mass $(\ln M)$ on body length $(\ln L)$. Genetic effects $(G)$ on condition were tested (main effect plus interaction with body length) with an ANCOVA $(\ln M-\ln L+$ $G+\ln L * G$ ), using for $G$ either genotype as a factor, or number of 227 copies as a covariate. Statistical analyses were performed using R 2.6.1 ( $\mathrm{R}$ development Core Team 2007).

The published forward and reverse Ha5-22 primers (Arens et al. 2000) delimit a ca. 250 bp region of genomic DNA. PCR-products were cloned with the pGEMeasy cloning kit (Promega) using the protocol described in Stöck et al. (2008). Twelve clones each from three individuals were sequenced in both directions (one female 227/227, one female 227/230, and one male 227/236). Clones were aligned with the program Sequencher 4.7, and contigs of each allele were screened for singletons considered to result from PCR error.

\section{Results}

Altogether 245 adult males and 86 adult females were sampled. Genotypes frequencies per year and per populations are provided in Table 1. Allele 236 was fixed on the $\mathrm{Y}$ chromosome in both populations $(\mathrm{n}=245$ copies of $\mathrm{Y}$ chromosomes). Allele 227 segregated with allele 230 on

Table 1 Ha5-22 genotype frequencies per year and per population. For females, expected values assuming Hardy-Weinberg equilibrium are provided in brackets, and significance for $\mathrm{Chi}^{2}$ tests are given (NS = non significant)

\begin{tabular}{|c|c|c|c|c|}
\hline & Cheylas 2007 & Cheylas 2008 & $\begin{array}{l}\text { Laissaud } \\
2008\end{array}$ & Total \\
\hline 우 227/227 & $0.00(0.10)$ & $0.02(0.05)$ & $0.17(0.15)$ & $0.05(0.08)$ \\
\hline ㅇ $227 / 230$ & $0.58(0.43)$ & $0.39(0.35)$ & $0.55(0.47)$ & $0.46(0.39)$ \\
\hline 우 230/230 & $\begin{array}{l}0.42(0.47) \\
n=19\end{array}$ & $\begin{array}{l}0.59(0.60) \\
n=49\end{array}$ & $\begin{array}{l}0.28(0.38) \\
n=18\end{array}$ & $\begin{array}{l}0.49(0.53) \\
n=86\end{array}$ \\
\hline $\mathrm{Chi}^{2}$ & $2.94 \mathrm{NS}$ & $1.10 \mathrm{NS}$ & $0.76 \mathrm{NS}$ & $2.71 \mathrm{NS}$ \\
\hline ô $227 / 236$ & 0.36 & 0.23 & 0.35 & 0.27 \\
\hline ô $230 / 236$ & $\begin{array}{l}0.64 \\
n=22\end{array}$ & $\begin{array}{l}0.77 \\
n=158\end{array}$ & $\begin{array}{l}0.65 \\
n=65\end{array}$ & $\begin{array}{l}0.73 \\
n=245\end{array}$ \\
\hline
\end{tabular}

Table 2 Allelic frequencies of Ha5-22 X-linked allele 227 per sex and per population. Sample sizes (number of X-chromosomal copies) and significance for $\mathrm{Chi}^{2}$ tests are given (NS = non significant)

\begin{tabular}{llll}
\hline & Cheylas & Laissaud & Both populations \\
\hline \multirow{0}{*}{$+{ }^{2}$} & $0.244(n=180)$ & $0.350(n=65)$ & $0.273(n=245)$ \\
+ & $0.235(n=136)$ & $0.440(n=36)$ & $0.279(n=172)$ \\
Chi $^{2}$ & $0.04 \mathrm{NS}$ & $0.80 \mathrm{NS}$ & $0.02 \mathrm{NS}$ \\
\hline
\end{tabular}

the $\mathrm{X}$ chromosome at an average frequency $\mathrm{q}=0.276$ ( $\mathrm{n}=417$ copies of the $\mathrm{X}$ chromosomes). Frequencies differed between sites in $2008\left(\mathrm{Chi}^{2}=9.85, P<0.005\right)$, being higher in Laissaud $(\mathrm{q}=0.386)$ than in Cheylas $(\mathrm{q}=0.223)$, but did not differ between years in Cheylas $\left(\mathrm{Chi}^{2}=2.35, P>0.1\right)$. Average frequency of allele 227 did not differ between males and females (Table 2). Female genotype frequencies displayed a slight but nonsignificant excess of heterozygotes ( 40 observed vs. 33.72 expected, $\mathrm{n}=86$ females, $\mathrm{Chi}^{2}=2.70, P=0.1$ ).

We analyzed 1,136 offspring (summed over years and populations, and including both tadpoles and froglets) from 44 families, classified in three groups according to parental Ha5-22 genotypes (Table 3). The first group (A) comprised 11 families with 227/230 mothers and 227/236 fathers. The 474 offspring genotyped from these families displayed a significant deficit of females 227/227 and 227/230, compared to expected Mendelian frequencies of $25 \%$ for each of the genotypes $\left(\mathrm{Chi}^{2}=6.22, P<0.05\right)$, resulting in a slightly male-biased sex-ratio $(56 \%)$. The second group (B) comprised 11 families with 227/230 mothers and 230/236 fathers. The 192 offspring genotyped displayed a significant deficit in $227 / 230$ females $\left(\mathrm{Chi}^{2}=9.54\right.$, $P<0.01$ ), also resulting in a slightly male biased sex-ratio $(56 \%)$. Finally, the third set (C), used as control, comprised 22 families with 230/230 mothers and 230/236 fathers. The 470 offspring analyzed displayed an even sex ratio (49\%) with no significant departure from Mendelian frequencies $\left(\mathrm{Chi}^{2}=0.08\right.$, NS $)$. Combining these results confirms that numbers differed from Mendelian expectations $\left(\mathrm{Chi}^{2}=\right.$ 11.04, $P<0.01$; Table 3), mostly due to a deficit in $227 / 227$ and 227/230 females, and a slight excess of $227 / 236$ males. Unfortunately, we could not obtain offspring from 227/227 females, because such genotypes are very scarce and pairs were crossed in the field without prior information about their genotypes.

Following Rice (1984), a mutation (227) beneficial to the heterogametic sex but detrimental to the homogametic sex can spread provided $S>\frac{2 h T}{1-h T}$, where $S=\Delta_{227 / 236}$ is the fitness advantage of $227 / 236$ males, $T=-\Delta_{227 / 227}$ the fitness reduction of homozygous 227/227 females, and $h T=-\Delta_{227 / 230}$ that of heterozygous (227/230) females. From our data (Table 3), these parameters amount to $S=0.13, T=0.13$ and $h T=0.18$, so that conditions for a 
Table 3 Genotype frequencies among offspring from (A) 11 families with 227/230 mothers and 227/236 fathers, (B) 11 families with $227 / 230$ mothers and a 230/236 fathers, and (C) 22 families with 230/230 mothers and 230/236 fathers

\begin{tabular}{|c|c|c|c|c|}
\hline & $\begin{array}{l}\text { A } \\
\text { क } 227 / 230 \times \text { ô } 227 / 236\end{array}$ & $\begin{array}{l}\text { B } \\
\text { q } 227 / 230 \times \text { ô } 230 / 236\end{array}$ & $\begin{array}{l}\mathrm{C} \\
\text { क } 230 / 230 \times \text { ô } 230 / 236\end{array}$ & Total \\
\hline 우 227/227 & $0.22(0.25)$ & - & - & $0.09(0.11)$ \\
\hline q $227 / 230$ & $0.22(0.25)$ & $0.15(0.25)$ & - & $0.12(0.15)$ \\
\hline ㅇ $230 / 230$ & - & $0.29(0.25)$ & $0.51(0.5)$ & $0.26(0.25)$ \\
\hline ô 227/236 & $0.28(0.25)$ & $0.30(0.25)$ & - & $0.17(0.14)$ \\
\hline \multirow[t]{2}{*}{ స̃ $230 / 236$} & $0.28(0.25)$ & $0.26(0.25)$ & $0.49(0.5)$ & $0.36(0.35)$ \\
\hline & $n=476$ & $n=192$ & $n=470$ & $n=1136$ \\
\hline $\mathrm{Chi}^{2}$ & $6.22 *$ & $9.54 * *$ & $0.08 \mathrm{NS}$ & $11.04 * *$ \\
\hline
\end{tabular}

Expected values assuming Mendelian segregation are provided in brackets. Significance for $\mathrm{Chi}^{2}$ tests are given $[N S$ non significant; * Significant $(P<0.05)$; ** Highly significant $(P<0.01)]$

spread of allele 227 are not fulfilled. The strong counterselection in females should quickly eliminate allele 227 if such values were to apply under natural settings.

The genotypes of 46 tadpoles from the A group that died before metamorphosis did not differ from random regarding their Ha5-22 complement $\left(\mathrm{Chi}^{2}=0.26\right.$, NS). In addition, the body condition of 46 froglets from this same group, measured from the ANCOVA of $\ln$ (wet mass) against $\ln$ (body length), was not affected by their Ha5-22 genotype. Wet mass was largely explained by length $\left(r^{2}=0.88\right)$, but genotype affected neither the intercept nor the slope of the regression.

Sequence data fully confirmed the genotyping results. All sequenced clones of allele 227 (representing four allelic copies stemming from two females and one male) were identical in their $5-22_{\text {forw/rev }}$ regions, and differed from allele 230 by only one CAA (glutamine) deletion within the polyQ central region of the protein.

\section{Discussion}

Laboratory data provided somewhat conflicting results. On the one hand, genotypic frequencies within families differed significantly from Mendelian proportions. This discrepancy might stem either from meiotic drive, or, given that tadpoles were genotyped at a late stage (Gosner stage 30-36; Gosner 1960), more likely from genotypic differences in mortality during embryonic development (many clutches contained some eggs that failed to develop; E. Luquet and S. Plenet, personal observation). Accordingly, sex ratios were female-biased in offspring from 227/230 mothers (but not from the control 230/230 mothers), due to a deficit in 227/227 and 227/230 genotypes. At a first glance, therefore, patterns of early mortality seem consistent with a balanced selection stemming from sexantagonistic effects of allele 227 , positive in males, and negative in females.
On the other hand, however, no such genotypic effects could be detected later in development, either on tadpole survival or on froglet body condition. Furthermore, quantitative estimates of genotypic excesses or deficits did not fit the range of fitness differentials required for polymorphism maintenance [Eq. 3-5 in Rice (1984)]. Given estimated values, allele 227 should be quickly eliminated from populations, being strongly counter-selected in females. Assuming a role for female preference would not help, and actually further reinforce selective pressure against 227 , since females from male-heterogametic systems are expected to prefer mates carrying alleles beneficial to daughters (Albert and Otto 2005).

Field data also argue against a role for sex-antagonistic selection. Female genotypes did not depart significantly from Hardy-Weinberg equilibrium, and therefore do not support expectations of heterozygote-driven balancing selection. Similarly, the frequency of allele 227 did not differ between adult males and females, which is inconsistent with expectations from sex-antagonistic effects (Eq. 4 in Rice 1984). In both cases, significant deviations from HWE would be expected, if similar effects as those documented in the laboratory were also found under field conditions. To detect weaker selection, dataset for adults larger than ours ( 245 males, 86 females) would have been required to provide enough statistical power. Similarly, larger sample sizes in the family dataset (especially from families with 227/230 mothers and 230/236 fathers, Table 3) might have increased our power to estimate possible selective pressures on allele 227.

Early-mortality effects suggest some genotype-environment interactions. Laboratory rearing conditions differed from natural settings. Temperature, for instance, which is known to interact with embryonic development, was markedly higher than normally in the field. Fluctuation of environmental conditions within or across seasons at natural breeding sites might influence these interactions, and cause rapid re-establishment of HWE for Ha5-22 
genotypes. This might also explain our failure to detect signatures of selection. We cannot exclude, therefore, the possibility that allele 227 is counter-selected in females under conditions outside the natural range of this study (a point which might be clarified by additional experiments). However, our present results clearly do not support a role for sex-antagonistic selection in maintaining this X-linked polymorphism, which seems therefore neutral under current field conditions. The data suggest that the single glutamine deletion in the Ha5-22 allele 227 is insufficient to induce any functional changes that cause fitness differences as compared to $\mathrm{X}$-chromosomal allele 230. MED15 could just happen to be trapped in the non-recombining segment of the nascent sex chromosomes in $H$. arborea without having a sex-specific function. In this case, neutral transmission of different $\mathrm{X}$ and $\mathrm{Y}$-linked alleles would be expected.

Alternatively, sex-antagonistic effects might still account for the fixation of different alleles on the $\mathrm{X}$ and $\mathrm{Y}$ chromosomes. Whether allele 236 (found here to be fixed on the $\mathrm{Y}$, but absent from the $\mathrm{X}$, and differing from allele 230 by six base pair indels) has male-beneficial effects on fitness, remains also an open question, which might be similarly tested, provided relevant polymorphism on the $\mathrm{Y}$ chromosome can be found.

Acknowledgments P. Joly, J. P. Léna, J. Prunier, J. Chiaffi, D. Serol, C. Vialet and R. Gautron provided much-welcome help for the fieldwork, R. Sermier and K. Ghali for the lab work. This study was supported by the Swiss National Science Foundation for Scientific Research (Grant 3100A0-108100 to NP) and the French National Research Agency (ANR Grant COLAPSE BLAN06-1_158236 to Pierre Joly).

\section{References}

Albert, A. Y. K., \& Otto, S. P. (2005). Sexual selection can resolve sex-linked sexual antagonism. Science, 310, 119-121.

Arens, P., Van't Westende, W., Bugter, R., Smulders, M. J. M., \& Vosman, B. (2000). Microsatellite markers for the European tree frog Hyla arborea. Molecular Ecology, 9, 1944-1946.

Bachtrog, D. (2004). Evidence that positive selection drives Y-chromosome degeneration in Drosophila miranda. Nature Genetics, $36,518-522$.

Berset-Brändli, L., Jaquiery, J., Dubey, S., \& Perrin, N. (2006). A sexspecific marker reveals male heterogamety in European tree frogs. Molecular Biology and Evolution, 23, 1104-1106.

Berset-Brändli, L., Jaquiery, J., \& Perrin, N. (2007). Recombination is suppressed and variability reduced in a nascent $\mathrm{Y}$ chromosome. Journal of Evolutionary Biology, 20, 1182-1188.

Broquet, T., Berset-Brändli, L., Emaresi, G., \& Fumagalli, L. (2007). Buccal swabs allow efficient and reliable microsatellite genotyping in amphibians. Conservation Genetics, 8, 509-511.

Buchanan, G., Yang, M., Cheong, A., Harris, J. M., Irvine, R. A., Lambert, P. F., et al. (2004). Structural and functional consequences of glutamine tract variation in the androgen receptor. Human Molecular Genetics, 13, 1677-1692.
Charlesworth, B. (1991). The evolution of sex-chromosomes. Science, 251, 1030-1033.

Charlesworth, B. (2002). The evolution of chromosomal sex determination. In D. Chadwick \& J. Goode (Eds.), The genetics and biology of sex determination: Novartis foundation symposium 244 (pp. 207-224). Chichester: John Wiley \& Sons Ltd.

Charlesworth, B. (2004). Sex determination: Primitive Y chromosomes in fish. Current Biology, 14, R745-R747.

Charlesworth, B., \& Charlesworth, D. (2000). The degeneration of Y chromosomes. Philosophical Transactions of the Royal Society of London Series B-Biological Sciences, 355, 1563-1572.

Filatov, D. A., Moneger, F., Negrutiu, I., \& Charlesworth, D. (2000). Low variability in a Y-linked plant gene and its implications for Y-chromosome evolution. Nature, 404, 388-390.

Gerber, H. P., Seipel, K., Georgiev, O., Hofferer, M., Hug, M., Rusconi, S., et al. (1994). Transcriptional activation modulated by homopolymeric glutamine and proline stretches. Science, 263, 808-811.

Gibson, J. R., Chippindale, A. K., \& Rice, W. R. (2002). The X chromosome is a hot spot for sexually antagonistic fitness variation. Proceedings of the Royal Society of London Series B-Biological Sciences, 269, 499-505.

Gosner, K. L. (1960). A simplified table for staging anuran embryos and larvae with notes on identification. Herpetologica, 16, 8.

Haldane, J. B. S. (1922). Sex ratio and unisexual sterility in hybrid animals. Journal of Genetics, 12, 101-109.

Hancock, J. M., Worthey, E. A., \& Santibanez-Koref, M. F. (2001). A role for selection in regulating the evolutionary emergence of disease-causing and other coding CAG repeats in humans and mice. Molecular Biology and Evolution, 18, 1014-1023.

Kondo, M., Nanda, I., Hornung, U., Schmid, M., \& Schartl, M. (2004). Evolutionary origin of the medaka Y chromosome. Current Biology, 14, 1664-1669.

Liu, Z. Y., Moore, P. H., Ma, H., Ackerman, C. M., Ragiba, M., Yu, Q. Y., et al. (2004). A primitive Y chromosome in papaya marks incipient sex chromosome evolution. Nature, 427, $348-352$.

McAllister, B. F., \& Charlesworth, B. (1999). Reduced sequence variability on the neo-Y chromosome of Drosophila americana americana. Genetics, 153, 221-233.

Mularoni, L., Veitia, R. A., \& Alba, M. M. (2007). Highly constrained proteins contain an unexpectedly large number of amino acid tandem repeats. Genomics, 89, 316-325.

Nanda, I., Kondo, M., Hornung, U., Asakawa, S., Winkler, C., Shimizu, A., et al. (2002). A duplicated copy of DMRT1 in the sex-determining region of the Y chromosome of the medaka, Oryzias latipes. Proceedings of the National Academy of Sciences of the United States of America, 99, 11778-11783.

Nei, M. (1969). Linkage modification and sex differences in recombination. Genetics, 63, 681-699.

Niculita-Hirzel, H., Stöck, M., \& Perrin, N. (2008). A key transcription cofactor on the nascent sex chromosomes of European tree frogs (Hyla arborea). Genetics, 179, 1721-1723.

Peichel, C. L., Ross, J. A., Matson, C. K., Dickson, M., Grimwood, J., Schmutz, J., et al. (2004). The master sex-determination locus in threespine sticklebacks is on a nascent $\mathrm{Y}$ chromosome. Current Biology, 14, 1416-1424.

Perutz, M. F., Johnson, T., Suzuki, M., \& Finch, J. T. (1994). Glutamine repeats as polar zippers-their possible role in inherited neurodegenerative diseases. Proceedings of the National Academy of Sciences of the United States of America, 91, 5355-5358.

Pidancier, N., Miquel, C., \& Miaud, C. (2003). Buccal swabs as a non-destructive tissue sampling method for DNA analysis in amphibians. Herpetological Journal, 13, 175-178. 
R Development Core Team. (2007). R: A language and environment for statistical computing. Vienna, Austria: R Foundation for Statistical Computing. http://www.R-project.org.

Rice, W. R. (1984). Sex-chromosomes and the evolution of sexual dimorphism. Evolution, 38, 735-742.

Rice, W. R. (1987). The accumulation of sexually antagonistic genes as a selective agent promoting the evolution of reduced recombination between primitive sex-chromosomes. Evolution, 41, 911-914.

Rice, W. R., \& Chippindale, A. K. (2001). Intersexual ontogenetic conflict. Journal of Evolutionary Biology, 14, 685-693.
Shimohata, T., Nakajima, T., Yamada, M., Uchida, C., Onodera, O., Naruse, S., et al. (2000). Expanded polyglutamine stretches interact with TAF(II)130, interfering with CREB-dependent transcription. Nature Genetics, 26, 29-36.

Steinemann, S., \& Steinemann, M. (2005). Y chromosomes: Born to be destroyed. Bioessays, 27, 1076-1083.

Stöck, M., Sicilia, A., Belfiore, N. M., Buckley, D., Lo Brutto, S., Lo Valvo, M., et al. (2008). Post-Messinian evolutionary relationships across the Sicilian channel: Mitochondrial and nuclear markers link a new green toad from Sicily to African relatives. BMC Evolutionary Biology, 8, 56. 